\title{
Predictive Value of Gut Microbiome for Cognitive Impairment in Patients with Hypertension
}

\author{
Shourong Lu, ${ }^{1}$ Lin Shao, ${ }^{2}$ Yunyun Zhang, ${ }^{3}$ Ying Yang, ${ }^{1}$ Zhuo Wang, ${ }^{1}$ Bingshan Zhang, \\ Jie Yu, ${ }^{1}$ Qiao Xu, ${ }^{1}$ Shuqiang Wang, ${ }^{4}$ Xiaorong Chen, ${ }^{4}$ Zhiming Yu ${ }^{(D},{ }^{5}$ Yilin Ren ${ }^{D},{ }^{6}$ \\ and Kan Hong ${ }^{1}{ }^{1}$ \\ ${ }^{1}$ Department of Geriatric, The Affiliated Wuxi People's Hospital of Nanjing Medical University, Wuxi 214000, China \\ ${ }^{2}$ Department of Radiology, The Affiliated Hospital of Jiangnan University, Wuxi 214000, China \\ ${ }^{3}$ Department of General Practice, The Affiliated Wuxi People's Hospital of Nanjing Medical University, Wuxi 214000, China \\ ${ }^{4}$ Department of Medicine, Wuxi Xin'an Community Health Service Center, Wuxi 214135, China \\ ${ }^{5}$ Department of Cardiovascular, The Affiliated Wuxi People's Hospital of Nanjing Medical University, Wuxi 214000, China \\ ${ }^{6}$ School of Pharmaceutical Sciences, Jiangnan University, Wuxi 214122, China
}

Correspondence should be addressed to Zhiming Yu; yuzhimi8660@sina.com, Yilin Ren; elyn0108@163.com, and Kan Hong; lindashao99@163.com

Received 10 August 2021; Revised 6 September 2021; Accepted 9 September 2021; Published 6 October 2021

Academic Editor: Yuzhen Xu

Copyright (c) 2021 Shourong Lu et al. This is an open access article distributed under the Creative Commons Attribution License, which permits unrestricted use, distribution, and reproduction in any medium, provided the original work is properly cited.

\begin{abstract}
A connection exists between hypertension (HTN) and cognitive impairment (CI) or gut microbiota (GM) and neuropsychiatric disease. However, the link between GM and HTNCI has not been illustrated. This study endeavoured to profile the landscape of GM in HTNCI patients and evaluate the value of GM as HTNCI biomarkers. We recruited 128 patients with hypertension and assigned them to two groups of different MoCA scores. Clinical and biological data were recorded. GM composition was illustrated with $16 \mathrm{~S}$ ribosomal RNA sequencing, and the dominant species were identified by linear discriminant analysis Effect Size (LEfSe). It showed higher abundance of TM7 and lower abundances of Veillonella and Peptoniphilus in the HTNCI group than in the HTN without cognitive impairment (HTNnCI) group. We next clarified the link between GM and MoCA scores or HTNCI factors. KEGG analysis revealed the involvement of decreased bile secretion. An evident correlation showed up between HTNCI and Veillonella abundance $(P=0.0340)$. We concluded that some representative GM species, especially Veillonella, could predict cognitive impairment in hypertension patients, making them potential benchmarks of HTNCI.
\end{abstract}

\section{Introduction}

Dementia, often aging-related, features progressive and irreversible cognitive decline severe enough to impair the quality of life [1]. About 50 million people are suffering worldwide, with an expected annual increase of 9.9 million cases [2]. Hypertension (HTN), a culprit of cognitive impairment, is confirmed in many studies [3-7]. According to the plan of the World Health Organization, a 25\% reduction in HTN prevalence by 2025 is a key effort to control cognitive decline [2]. Hypertension boosts arteriosclerotic progression in the brain, facilitating the atheroma formation and arteriolar tortuosity $[8,9]$. Hypoperfusion can bring with infarction and diffuse ischemia in the periventricular and deep white matter, which favours the development of Alzheimer's disease (AD) [9]. As two leading causes of cognitive impairment, $\mathrm{AD}$ is manifested by early loss of episodic memory and vascular cognitive impairment (VCI) by impairment inattention, information processing, and executive function [10].

The human gastrointestinal tract populates a colony of symbiotic bacteria with abundant species and a dynamic balance. Gut microbiota (GM) is tightly involved in the physiology and pathology of humans [11]. In recent years, the imbalance of GM and its products is importantly related to the occurrence of diseases such as obesity, diabetes, 
hyperlipidemia, and hypertension [12-14]. In patients with blood pressure and cognitive impairment, the GM affects the human blood pressure level, brain function, and host behavior, which is related to hypertension with cognitive impairment. Studies have shown that regulating the GM can improve hypertension [15], becoming a potential key target for improving hypertension with cognitive impairment.

GM dysbiosis has a link with neuropsychiatric disorders in human and animal studies. Studies have shown characteristics of fecal microbial diversity and composition in $\mathrm{AD}$ patients: more bacteria eliciting proinflammatory response and less bacteria synthesizing short-chain fatty acids (SCFAs) $[16,17]$. In an AD mouse model, Verrucomicrobia and Proteobacteria increase, and Ruminococcus and Butyricicoccus decrease [18]. Moreover, the tie between GM and cognitive impairment has been clarified in other diseases [19-22]. However, the profile of GM in HTNCI patients has not been elucidated. The association between GM and brain function has been explored in models using germfree mice or animals treated with probiotics. In these studies, germ-free mice demonstrated aberrant social behaviors [23, 24] and alterations in the amygdala and prefrontal cortex $[25,26]$. Other experiments based on germ-free animals found that GM was closely implicated in neurogenesis, a process critical to learning and memory [27]. Probiotics alleviated anxiety and depression in rats and mice [28]. Oral SCFAs also inhibited the decline in the function of microglia in germ-free animals [29]. Fecal microbiota transplantation could produce behavioral phenotypes [30]. However, it is a question whether these outcomes in animal experiments can be achieved in humans.

Here, we characterized the GM profile and its link with MoCA scores and HTNCI risk factors. Our findings may pluck potential diagnostic biomarkers out of GM for HTNCI.

\section{Materials and Methods}

2.1. Study Patients. Patients, aged $>60$ years, confirmed with hypertension, and treated in the Xin'an community in Wuxi city from May to October 2018 were enrolled. The exclusion criteria included the following: severe vision, hearing and speaking impairments, use of antibiotics or probiotics within the previous 6 months, diet restriction, gastrointestinal surgery, infection, mental disorders (such as schizophrenia), and severe life-threatening illnesses. The study protocol was approved by the Ethics Committee of the Wuxi People's Hospital. Each patient provided written informed consent.

2.2. Neuropsychological Assessment. The neuropsychological function was scored by the Montreal Cognitive Assessment (MoCA) (https://www.mocatest.org/) at 3 months after hypertension onset. A score $\geq 26$ was defined as normal. To control the bias in MoCA assessment due to the education background of the patient, one point was given to the patient having education of $<12$ years, but not if the total score of this patient exceeded 30 points.

2.3. Clinical Data Collection. Basic information was collected during the clinical interview, including sex, age, education level, physique, sleep, smoking and alcohol intake, dietary habit, body mass index (BMI), and profiles of vitamin B12 and thyroid-stimulating hormone (TSH). We also prepared 128 fecal samples and stored them at $-80^{\circ} \mathrm{C}$.

2.4. Bioinformatics and Data Analysis. DNA extraction from fecal samples was performed with the FastDNA Spin Kit (MP Biomedicals, Santa Ana, CA, USA), followed by amplification of V3-V4 16S ribosomal RNA with the primers $5^{\prime}$-CTCCTACGGGAGGCAGCA-3' and $5^{\prime}$-GGACTACHVGGGTWTCTAAT- ${ }^{\prime}$. Sequencing was conducted on an Illumina MiSeq PE300 platform (Illumina, Santiago, CA, USA). After analysis on the QIIME pipeline, only high-quality sequences were retained (score $>30$ and length $\geq 200 \mathrm{bp}$ ). Those with $>97 \%$ similarity were concentrated into operational taxonomic units (OTUs) by QIIME 1.9.1 (https://qiime.org/), followed by the generation of taxonomic profiles of each OTU at five levels (phylum, class, order, family, and genus). Principal component analysis was carried out on SIMCA 14.0 (Umetrics AB, Umeå, Sweden). Candidate biomarkers were filtered out through linear discriminant analysis Effect Size (LEfSe). With an alpha value of 0.05 for both the factorial Kruskal-Wallis test among classes and the pairwise Wilcoxon-Mann-Whitney test between subclasses, the threshold on the logarithmic LDA score for discriminative features was set at 2.0. Phylogenetic investigation of communities by reconstruction of unobserved states (PICRUSt) was employed to predict the gene function of gut microbiota between groups with and without cognitive impairment. Related pathways were determined using Kyoto Encyclopedia of Genes and Genomes (KEGG) Orthology. The processing of 16S rRNA sequencing data was implemented in LEfSe and PICRUSt online (https:// huttenhower.sph.harvard.edu/galaxy).

2.5. Statistical Analysis. Data were analyzed with GraphPad Prism V.7.0.1 (La Jolla, CA, United States), R software (V.3.4), and Adobe Illustrator CC 2015 (Adobe Systems Incorporated, California, America). Categorical variables were subjected to a chi-squared test and continuous variables to Student's $t$-test or Mann-Whitney test. The Mann-Whitney test was carried out to compare the data of the HTNCI and HTNnCI groups. Multivariate logistic regression was to determine the factors, especially representative microbes, for predicting HTNCI. The probability cutoff value was 0.05 to put in and 0.1 to put out a variable. Spearman's rank correlation was used to demonstrate the link of GM with MoCA scores and HTNCI risk factors.

\section{Results}

3.1. Baseline Patient Information. Recruited were 234 patients with hypertension. Excluded were 36 for unwillingness to join the study, 42 for missing data, and 28 for exclusion criteria. The 128 eligible were assigned to the HTNnCI group $(n=60)$ and the HTNCI group $(n=68)$ (see Figure 1). As shown in Table 1, significant differences were observed in sex, education level, MoCA score, stroke, and thyroidstimulating hormone between two groups $(P=0.0269$, 


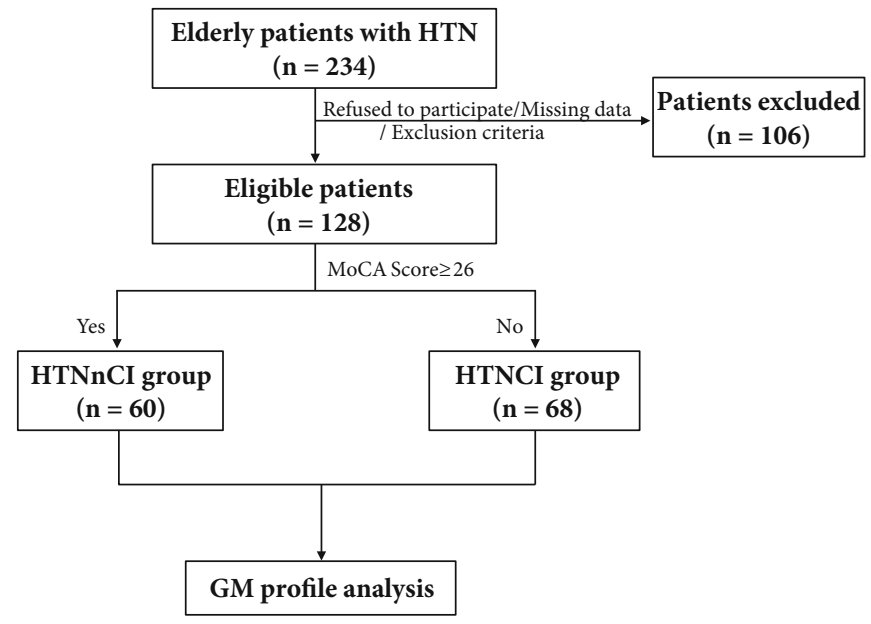

Figure 1: Flow chart of patient recruitment.

TABLE 1: Baseline information of the HTNCI and HTNnCI groups.

\begin{tabular}{lccc}
\hline Parameters & $\begin{array}{c}\text { HTNnCI group } \\
(n=60)\end{array}$ & $\begin{array}{c}\text { HTNCI group } \\
(n=68)\end{array}$ & $P$ value \\
\hline $\begin{array}{l}\text { Age (years) } \\
\text { Gender (male/ }\end{array}$ & 68.23 & 69.56 & 0.1233 \\
total\%) & 51.67 & 32.35 & 0.0269 \\
Low education & 2.43 & 2.15 & 0.0161 \\
BMI (kg/m $\left.{ }^{2}\right)$ & 24.75 & 25.40 & 0.2179 \\
$\begin{array}{l}\text { Smoking (still } \\
\text { smoking/total\%) }\end{array}$ & 30.00 & 25.00 & 0.6908 \\
$\begin{array}{l}\text { Alcohol (still } \\
\text { drinking/total\%) }\end{array}$ & 25.00 & 16.18 & 0.3591 \\
MoCA score & 27.27 & 19.16 & $<0.0001$ \\
$\begin{array}{l}\text { Diabetes mellitus } \\
\text { Coronary heart }\end{array}$ & 0.30 & 0.29 & 0.9426 \\
disease & 0.083 & 0.18 & 0.1232 \\
Stroke & 0.10 & 0.26 & 0.0191 \\
Vitamin B12 (pg/ & 263.00 & 230.50 & 0.3288 \\
mL) & 4.91 & 10.02 & 0.0253 \\
TSH (mU/L) & & & \\
\hline
\end{tabular}

TSH: thyroid-stimulating hormone.

$0.0161,<0.0001,0.0191$, and 0.0253 , respectively), but not in age, body mass index (BMI), smoking, alcohol use, diabetes mellitus, coronary heart disease, and vitamin B12.

3.2. GM Profile in HTNCI Patients. A total of 7825 OTUs were generated by $16 \mathrm{~S}$ ribosomal RNA sequencing, including 14 phyla, 22 classes, 29 orders, 64 families, and 151 genera. Fecal microbiota alpha-diversity columns and the PCoA scatterplots showed no compositional difference between groups, but significant differences in the diversity of some taxa (see Figure 2).

At the phylum level, the HTNCI group showed higher abundance of TM7 (0.00992647 vs. $0.0142756 \%, P=0.014$, see Figure 3(a)) and lower abundance of Synergistetes ( 0.0456658 vs. $0.00105054 \%, P=0.049$, see Figure 3(a)). As to classes, the HTNCI group showed higher abundance of TM7-3 (0.009926 vs. $0.014275632 \%, P=0.014$, see Figure 3(b)). At the genus level, the HTNCI group showed higher abundances of 6 genera, including Paludibacter $(0.000$ vs. $0.033162 \%, P<0.0001$, see Figure 3(c)), Acidaminococcus ( 0.002859 vs. $0.042649 \%, P<0.0001$, see Figure 3(c)), Morganella ( 0.003608 vs. $0.026179 \%, P<0.0001$, see Figure $3(\mathrm{c})$ ), Eubacterium $(0.002758$ vs. $0.013626 \%, P=0.0032$, see Figure 3(e)), S24-7 unclassified (0.008 vs. 0.00819\%, $P=$ 0.0370 , see Figure 3(e)), and Peptococcus ( 0.024134 vs. $0 \%, P$ $=0.0010$, see Figure 3(c)), and lower abundances of 17 genera, including Veillonella ( 0.186036 vs. $0.05164 \%, P=0.0340$, see Figure 3(c)), Christensenellaceae unclassified (0.019928 vs. $0.005697 \%, P<0.0001$, see Figure 3(c)), Anaerotruncus (0.006951 vs. $0.020673 \%, P=0.0001$, see Figure 3(c)), Citrobacter $(0.011582$ vs. $0.017372 \%, P=0.0044$, see Figure $3(\mathrm{c}))$, and TM7-3_unclassified ( 0.009562 vs. $0.014074 \%, P=0.0200$, see Figure 3(c)). In LEfSe analysis, the HTNCI group showed a higher abundance of Veillonella and a lower abundance of Bilophila (see Figures 3(d) and 3(e)).

3.3. Predicted Functions of GM. We evaluated the functional differences of GM between the HTNnCI and HTNCI groups. As shown in Figure 4, in the HTNCI group, the GM was enriched in bile secretion (organismal systems, digestive system), shigellosis (human diseases, infectious diseases), and $\mathrm{G}$ protein-coupled receptors (environmental information processing, signalling molecules, and interaction). In HTNnCI patients, the enrichments included photosynthesis-antenna proteins (metabolism, energy metabolism), betalain biosynthesis (metabolism, biosynthesis of other secondary metabolites), biosynthesis of type II polyketide products (metabolism, metabolism of terpenoids and polyketides), and melanogenesis (organismal systems, endocrine system).

3.4. Correlation between GM and MoCA Score and the Risk Factors for Cognitive Impairment. According to the results of Spearman's rank analysis (see Figure 5), Lachnospira $(P<0.05)$ Veillonella $\quad(P<0.05), \quad$ Firmicutes_Other 


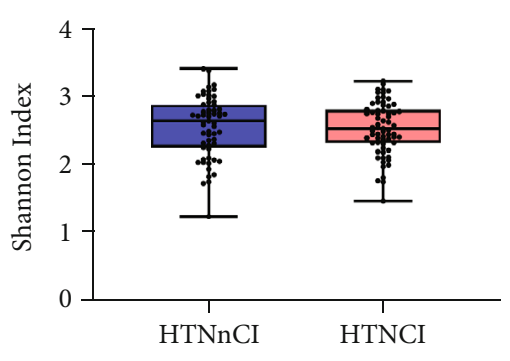

(a)

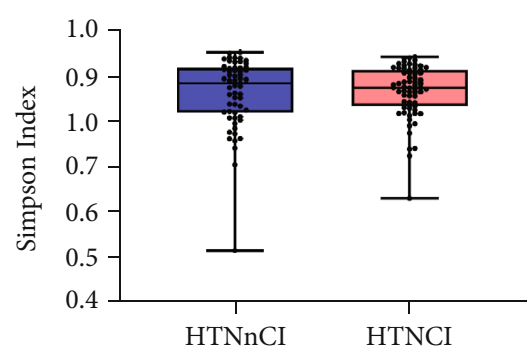

(b)

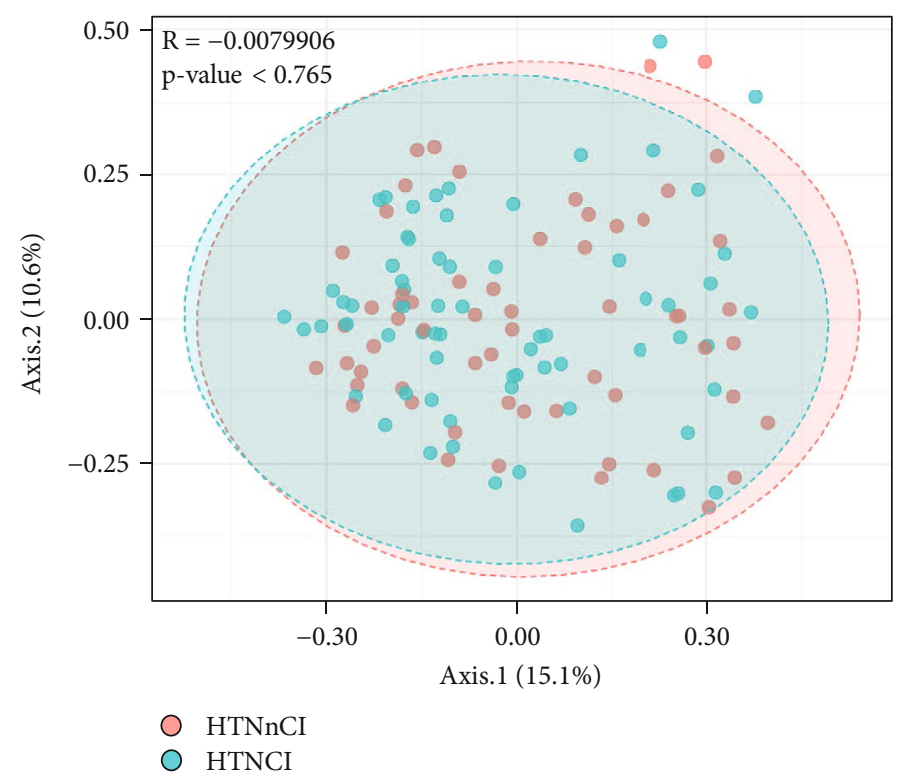

(c)

FIGURE 2: Comparison of GM components between groups' hypertension without cognitive impairment (HTNnCI) and hypertension with cognitive impairment (HTNCI). The Shannon (a) and Simpson (b) indexes were used to assess the alpha-diversity between the two groups. $P$ values were determined using Mann-Whitney $U$-test. (c) Scatterplot from principal coordinates analysis showed the similar distribution between the two groups.

$(P<0.01)$, and Peptoniphilus $(P<0.05)$ levels were positively associated with the MoCA score. Moreover, Paludibacter demonstrated a positive correlation with TSH $(P<0.05)$ and stroke $(P<0.05)$, but a negative correlation with vitamin $\mathrm{B}(P<0.05)$. Prevotella was negatively correlated with TSH $(P<0.05)$ and hypertension $(P<0.05)$. In addition, Methanobrevibacter was negatively associated with diabetes $(P<0.05)$ and hypertension $(P<0.05)$. Prevotella was negatively correlated with TSH $(P<0.05)$, while Clostridium $(P<0.01)$, Paludibacter $(P<0.05)$, Oscillospira $(P<0.05)$, and Sutterella $(P<0.05)$ were all positively associated with TSH. Taken together, GM might harbor biomarkers sensitive to HTNCI.

\section{Discussion}

We drew a profile of GM in HTNCI patients in the present study. GM exhibited no significant difference in GM diversity in HTNCI patients, but in abundances of various GM components. The low abundance of Veillonella was detected in the HTNCI group, and its potential to predict cognitive impairment was verified in LEfSe analysis. Moreover, we associated GM profile with MoCA score and HTNCI risk factors, such as education, sex, stroke, hypertension, diabetes, vitamin B12, and TSH. Interestingly, Veillonella also showed an ability to discriminate HTNCI. It is suggested that GM might contain efficient biomarkers for HTNCI.

In this study, sex, education, stroke, and TSH showed between-group differences and close associations with GM. Positive correlations between hypertension and cognitive impairment and GM dysbiosis have been determined [31, 32]. Besides, high TSH may increase blood pressure to facilitate cognitive impairment [33]. This mechanism may involve the upregulation of proinflammatory cytokines, endothelial damage, and subsequent neurotoxic effects [34]. Thyroid diseases, either subclinical or clinical, interact with cardiovascular disease. A connection has showed up between thyroid function and $\mathrm{AD}$ [35-38]. As a typical feature of $\mathrm{AD}, \beta$-amyloid mediates neurotoxicity through various mechanisms, such as inhibiting acetylcholine activity in the cortex and hippocampus $[39,40]$. It also shows that thyroid function changes with systemic oxidative stress 


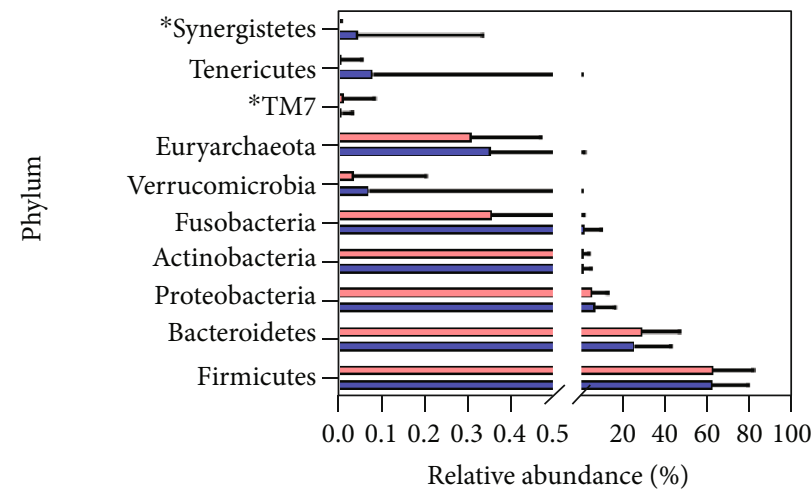

(a)

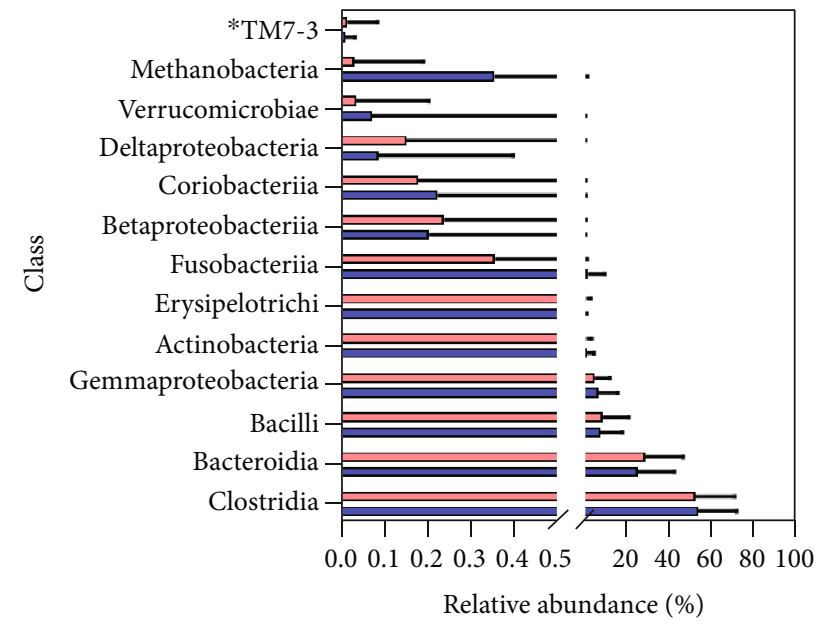

(b)

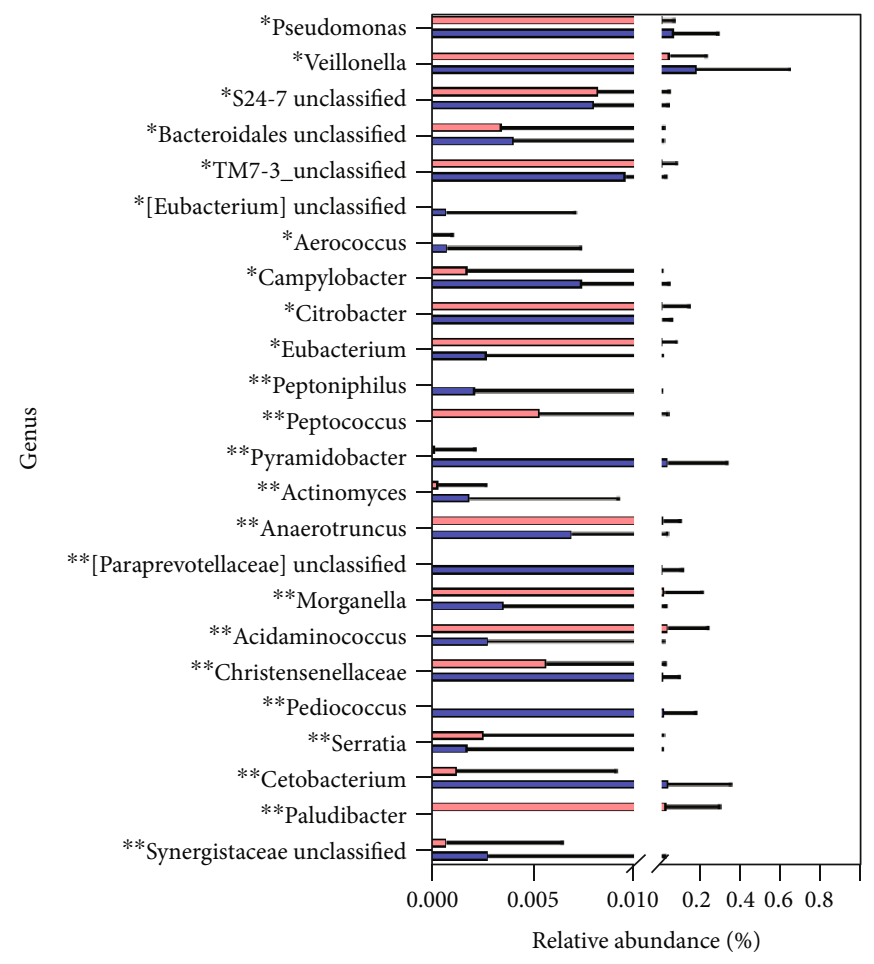

(c)

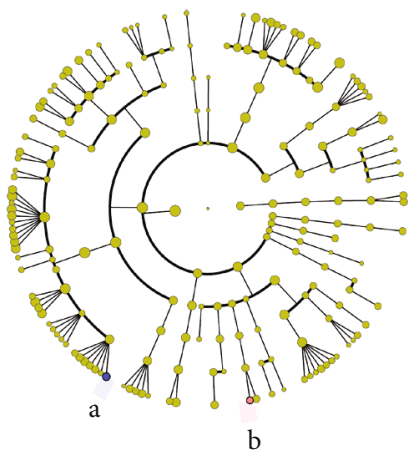

a: Veillonella

b: Bilophila

(d)

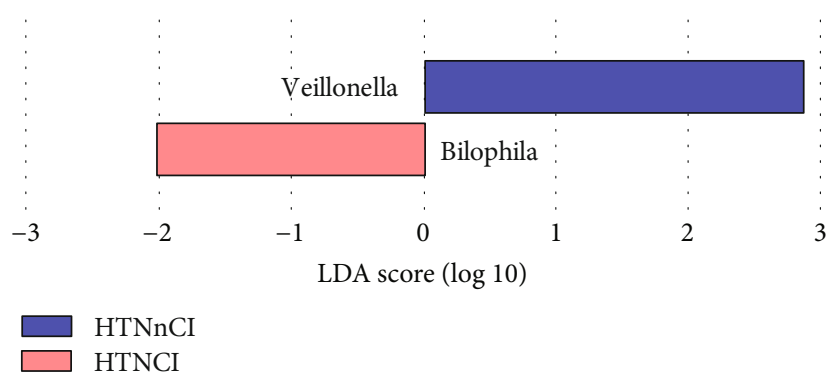

(e)

Figure 3: Comparison of abundances of representative GM species between two groups. The Mann-Whitney $U$-test indicated the significant between-group differences on phylum (a), class (b), and genus (c) levels. The Mann-Whitney $U$-test indicated significant between-group differences. ${ }^{*} P<0.05$; ${ }^{* *} P<0.01$. (d) A cladogram of different taxonomic components between two groups. (e) Linear discriminant analysis scores showed significant between-group differences. 


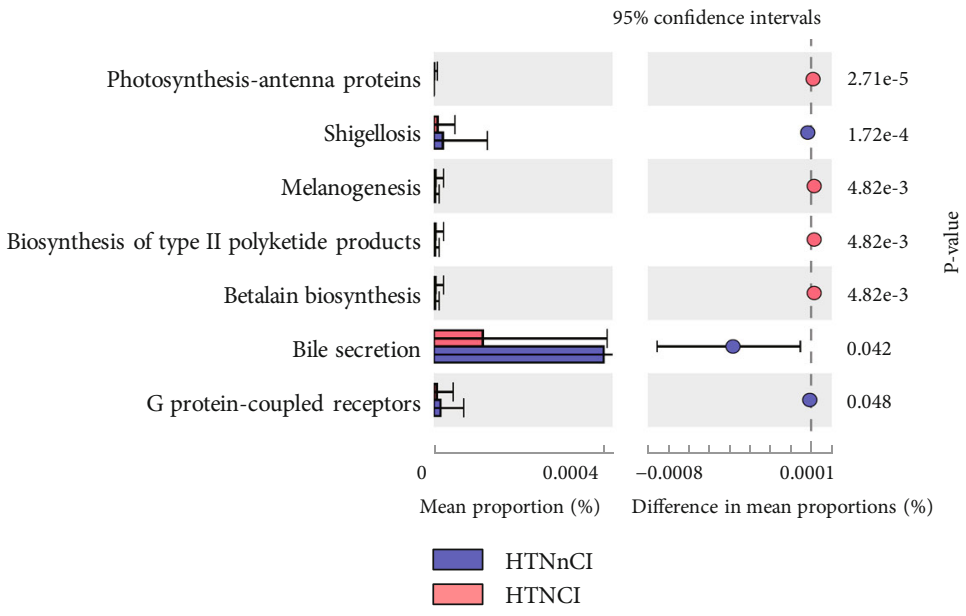

Figure 4: KEGG and GO analysis of gut microbiota in the HTNCI and HTNNCI groups.

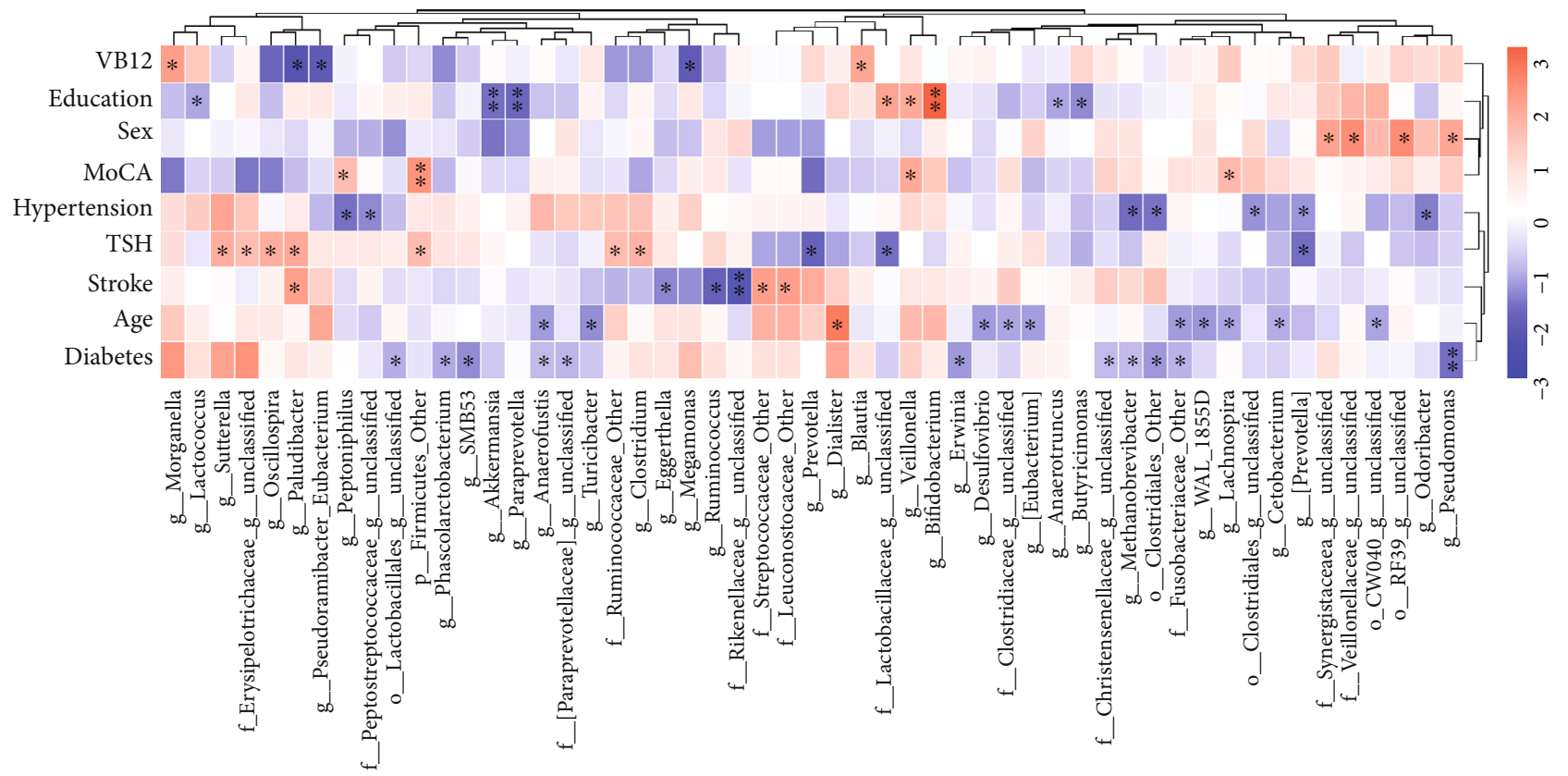

FIGURE 5: Heatmap of Spearman's rank correlation of gut microbiota with MoCA scores and risk factors for cognitive impairment. Red means positive correlation, and blue means negative correlation. ${ }^{*} P<0.05 ;{ }^{* *} P<0.01$.

[41]. Our results supported some epidemiological studies in which multiple factors associated with HTNCI had been identified, including TSH, stroke, and education.

A previous study has hinted that GM may be associated with hypertension [32, 42-44]. In this study, higher abundances of TM7 and TM7-3 were found in HTNCI patients than in HTNnCI patients. Another study has indicated that the enrichment of TM7 occurs with gut dysbiosis and contributes to inflammation [45]. Moreover, TM7 is associated with a compromised intestinal barrier and elevated intestinal immune infiltration [46]. Evidence supports that TM7 phyla and Proteobacteria participate in the pathogenesis of cognitive impairment $[47,48]$. Genera Paludibacter are positively associated with the course of Parkinson's disease [49], which is consistent with our study. Thus, all the evidences suggest the interplay between GM and HTNCI.
Besides, we presented that HTNCI involved several KEGG pathways. Bile secretion is progressively reduced in HTNCI patients. Cholesterol metabolism in the liver is closely linked with $\mathrm{AD}$ [50]. In fact, many genes responsible for cholesterol metabolism (e.g., BIN1 and CLU) contain susceptibility loci of $\mathrm{AD}[51,52]$. Cholesterol is decomposed by bile acids (BAs). Mounting evidence has associated immune dysregulation with $\mathrm{AD}$ pathology. Some immunerelated genes have been discovered as risk genetic variants in $\mathrm{AD}[51,53]$. These immune-related genes may drive $\mathrm{AD}$ through regulating BA metabolism or GM. For example, $\mathrm{ABI} 3$ and $\mathrm{MEF} 2 \mathrm{C}$ act in immune reaction upon proinflammatory stimuli from microbes $[54,55]$. There is growing evidence supporting the tight connection of intestinal microbiota with the performance of the central nervous system. The central and enteric nervous systems 
communicate through the "gut-brain metabolic axis," but the mechanisms hide to be clarified [56-58]. This axis is indispensable for metabolic pathways through which GM regulates metabolic activities [59]. Intestinal bacterial composition is associated with a gallery of neurological disorders [60-62]. Liver disease may aggravate cognitive dysfunctions, even leading to $\mathrm{AD}$ [63].

A link was set up between GM and MoCA scores or HTNCI factors. Spearman's analysis showed that Veillonella abundance rose as the MoCA score dropped. Previous studies have revealed that the abundance of Veillonella decreases in the case of cognitive impairment [64]. Veillonella species, as a harmless or even beneficial gram-negative anaerobic coccus, colonizes the mouth after one's birth [65]. Veillonella is also the main species in the gut to produce propionic acid in human GM [66]. In animal models of AD, SCFAs have shown abilities to enhance learning and memory function [67], exert neuroprotection and guard neuroplasticity, shrink $\beta$-amyloid plaques, and inactivate microglia [68]. Chen et al. have uncovered that fecal microbiota transplantation (FMT) could cure infarct and cerebral edema and rescue cognitive function in rats with ischemic stroke [69]. FMT can also raise the level of SCFAs, making it a potential treatment for $\mathrm{AD}$ [70]. However, more studies should be conducted to answer whether HTNCI arises from the scarcity of SCFAs-producing bacteria. Bilophila belongs to Desulfovibrionaceae, a type of proinflammatory bacteria that induce LPS accumulation during inflammatory response [71]. High-level Desulfovibrionaceae is also implicated in psychiatric disorders $[72,73]$, hinting at the association of Bilophila with HTNCI. This study provided that some species in GM could predict HTNCI, with an accuracy expected higher if combined with other valuable biomarkers.

Several limitations also exist in this study. Our results might be biased by some unstandardized variables, and the significance of the MoCA score might be overemphasized. Clinical scales should be employed to testify our results. In addition, we did not evaluate the GM profiles over a long period. Besides, the sample size was still not enough. Future experiments with larger gender-matched samples are needed.

The study has also its strengths. First, this is the first that reports the profile of the GM in HTNCI patients. Second, we constructed broader connections between GM and HTNCI risk factors. Third, this study laid a roadmap to explore reliable predictive biomarkers for HTNCI.

\section{Conclusion}

In summary, the abnormal structure of GM is associated with HTNCI. Some species in GM, especially Veillonella, might be adopted to predict HTNCI.

\section{Data Availability}

The analyzed data sets generated during the study are available from the corresponding authors on reasonable request.

\section{Ethical Approval}

The present study has been approved by the Committee on the Ethics of Clinical Experiments of the Wuxi People's Hospital (KS2019039).

\section{Conflicts of Interest}

The authors declare no conflicts of interest.

\section{Authors' Contributions}

Zhiming Yu and Yilin Ren performed the study conceptualization. Shourong $\mathrm{Lu}$ and Lin Shao performed the experiments and wrote the manuscript. Yunyun Zhang and Ying Yang performed the data analysis and interpretation. Zhuo Wang and Bingshan Zhang performed the data curation. Jie $\mathrm{Yu}$ and Qiao $\mathrm{Yu}$ contributed to the data acquisition and supervision. Shuqiang Wang and Xiaorong Chen performed the data validation. Kan Hong developed the experimental design and revised the manuscript. Shourong Lu and Lin Shao contributed equally to this work.

\section{Acknowledgments}

This work was funded by the young project of Wuxi Health Committee (Q201914), the Top Talent Support Program for Young and Middle-Aged People of Wuxi Health (BJ2020008), and the major project of Wuxi Health Committee (Z202002).

\section{References}

[1] S. A. Gale, D. Acar, and K. R. Daffner, "Dementia," The American Journal of Medicine, vol. 131, no. 10, pp. 1161-1169, 2018.

[2] WHO Guidelines Approved by the Guidelines Review Committee, Risk Reduction of Cognitive Decline and Dementia: WHO Guidelines, World Health Organization, Geneva, 2019.

[3] P. B. Gorelick, A. Scuteri, S. E. Black et al., "Vascular contributions to cognitive impairment and dementia: a statement for healthcare professionals from the American Heart Association/American Stroke Association," Stroke, vol. 42, no. 9, pp. 2672-2713, 2011.

[4] G. Bakris and M. Sorrentino, "Redefining hypertension - assessing the new blood-pressure guidelines," The New England Journal of Medicine, vol. 378, no. 6, pp. 497-499, 2018.

[5] Y. Xing, Y. Sun, S. Wang, F. Feng, D. Zhang, and H. Li, "Nocturnal blood pressure rise as a predictor of cognitive impairment among the elderly: a retrospective cohort study," $B M C$ Geriatrics, vol. 21, no. 1, p. 462, 2021.

[6] X. Y. Chen, Y. Yang, Z. Y. Tang et al., “Association between hypertension with hyperhomocysteinemia and cognitive impairment in the Kailuan Community of China: a crosssectional study," Biomedical and Environmental Sciences, vol. 34, no. 7, pp. 557-561, 2021.

[7] D. Hughes, C. Judge, R. Murphy et al., “Association of blood pressure lowering with incident dementia or cognitive impairment: a systematic review and meta-analysis," JAMA, vol. 323, no. 19, pp. 1934-1944, 2020.

[8] L. Pantoni, "Cerebral small vessel disease: from pathogenesis and clinical characteristics to therapeutic challenges," The Lancet. Neurology, vol. 9, no. 7, pp. 689-701, 2010. 
[9] J. Birns and L. Kalra, "Cognitive function and hypertension," Journal of Human Hypertension, vol. 23, no. 2, pp. 86-96, 2009.

[10] J. T. O’Brien, T. Erkinjuntti, B. Reisberg et al., "Vascular cognitive impairment," The Lancet. Neurology, vol. 2, no. 2, pp. 8998, 2003.

[11] S. V. Lynch and O. Pedersen, "The human intestinal microbiome in health and disease," The New England Journal of Medicine, vol. 375, no. 24, pp. 2369-2379, 2016.

[12] M. C. Dao and K. Clément, "Gut microbiota and obesity: concepts relevant to clinical care," European Journal of Internal Medicine, vol. 48, pp. 18-24, 2018.

[13] M. R. Halawa, M. A. El-Salam, B. M. Mostafa, and S. S. Sallout, "The gut microbiome, Lactobacillus acidophilus; relation with type 2 diabetes mellitus," Current Diabetes Reviews, vol. 15, no. 6, pp. 480-485, 2019.

[14] M. He and B. Shi, "Gut microbiota as a potential target of metabolic syndrome: the role of probiotics and prebiotics," Cell \& Bioscience, vol. 7, no. 1, p. 54, 2017.

[15] S. Adnan, J. W. Nelson, N. J. Ajami et al., "Alterations in the gut microbiota can elicit hypertension in rats," Physiological Genomics, vol. 49, no. 2, pp. 96-104, 2017.

[16] Z. Q. Zhuang, L. L. Shen, W. W. Li et al., "Gut microbiota is altered in patients with Alzheimer's disease," Journal of Alzheimer's disease, vol. 63, no. 4, pp. 1337-1346, 2018.

[17] J. P. Haran, S. K. Bhattarai, S. E. Foley et al., "Alzheimer's disease microbiome is associated with dysregulation of the antiinflammatory P-glycoprotein pathway," MBio, vol. 10, no. 3, 2019.

[18] L. Zhang, Y. Wang, X. Xiayu et al., "Altered gut microbiota in a mouse model of Alzheimer's disease," Journal of Alzheimer's disease, vol. 60, no. 4, pp. 1241-1257, 2017.

[19] J. S. Bajaj, P. B. Hylemon, J. M. Ridlon et al., "Colonic mucosal microbiome differs from stool microbiome in cirrhosis and hepatic encephalopathy and is linked to cognition and inflammation," American Journal of Physiology. Gastrointestinal and Liver Physiology, vol. 303, no. 6, pp. G675-G685, 2012.

[20] A. L. Carlson, K. Xia, M. A. Azcarate-Peril et al., "Infant gut microbiome associated with cognitive development," Biological Psychiatry, vol. 83, no. 2, pp. 148-159, 2018.

[21] H. Gao, Q. Jiang, H. Ji, J. Ning, C. Li, and H. Zheng, “Type 1 diabetes induces cognitive dysfunction in rats associated with alterations of the gut microbiome and metabolomes in serum and hippocampus," Biochimica et Biophysica Acta, Molecular Basis of Disease, vol. 1865, no. 12, p. 165541, 2019.

[22] P. Liu, L. Wu, G. Peng et al., "Altered microbiomes distinguish Alzheimer's disease from amnestic mild cognitive impairment and health in a Chinese cohort," Brain, Behavior, and Immunity, vol. 80, pp. 633-643, 2019.

[23] R. D. Heijtz, S. Wang, F. Anuar et al., "Normal gut microbiota modulates brain development and behavior," Proceedings of the National Academy of Sciences of the United States of America, vol. 108, no. 7, pp. 3047-3052, 2011.

[24] K. M. Neufeld, N. Kang, J. Bienenstock, and J. A. Foster, "Reduced anxiety-like behavior and central neurochemical change in germ-free mice," Neurogastroenterology and Motility, vol. 23, no. 3, pp. 255-e119, 2011.

[25] R. M. Stilling, F. J. Ryan, A. E. Hoban et al., "Microbes \& neurodevelopment - Absence of microbiota during early life increases activity-related transcriptional pathways in the amygdala," Brain, Behavior, and Immunity, vol. 50, pp. 209220, 2015.

[26] A. E. Hoban, R. M. Stilling, F. J. Ryan et al., "Regulation of prefrontal cortex myelination by the microbiota," Translational Psychiatry, vol. 6, no. 4, article e774, 2016.

[27] E. S. Ogbonnaya, G. Clarke, F. Shanahan, T. G. Dinan, J. F. Cryan, and O. F. O'Leary, "Adult hippocampal neurogenesis is regulated by the microbiome," Biological Psychiatry, vol. 78, no. 4, pp. e7-e9, 2015.

[28] T. G. Dinan, C. Stanton, and J. F. Cryan, "Psychobiotics: a novel class of psychotropic," Biological Psychiatry, vol. 74, no. 10, pp. 720-726, 2013.

[29] D. Erny, A. L. Hrabě de Angelis, D. Jaitin et al., "Host microbiota constantly control maturation and function of microglia in the CNS," Nature Neuroscience, vol. 18, no. 7, pp. 965-977, 2015.

[30] S. M. Collins, Z. Kassam, and P. Bercik, "The adoptive transfer of behavioral phenotype via the intestinal microbiota: experimental evidence and clinical implications," Current Opinion in Microbiology, vol. 16, no. 3, pp. 240-245, 2013.

[31] H. C. Muela, V. A. Costa-Hong, M. S. Yassuda et al., "Hypertension severity is associated with impaired cognitive performance," Journal of the American Heart Association, vol. 6, no. $1,2017$.

[32] H. Li, B. Liu, J. Song et al., "Characteristics of gut microbiota in patients with hypertension and/or hyperlipidemia: a crosssectional study on rural residents in Xinxiang County, Henan Province," Microorganisms, vol. 7, no. 10, p. 399, 2019.

[33] D. Y. Gaitonde, K. D. Rowley, and L. B. Sweeney, "Hypothyroidism: an update," American Family Physician, vol. 86, no. 3, pp. 244-251, 2012.

[34] S. Annerbo and J. Lökk, "A clinical review of the association of thyroid stimulating hormone and cognitive impairment," ISRN Endocrinology, vol. 2013, Article ID 856017, 6 pages, 2013.

[35] L. A. van Osch, E. Hogervorst, M. Combrinck, and A. D. Smith, "Low thyroid-stimulating hormone as an independent risk factor for Alzheimer disease," Neurology, vol. 62, no. 11, pp. 1967-1971, 2004.

[36] A. E. Hak, H. A. Pols, T. J. Visser, H. A. Drexhage, A. Hofman, and J. C. M. Witteman, "Subclinical hypothyroidism is an independent risk factor for atherosclerosis and myocardial infarction in elderly women: the Rotterdam Study," Annals of Internal Medicine, vol. 132, no. 4, pp. 270-278, 2000.

[37] B. Biondi, E. A. Palmieri, G. Lombardi, and S. Fazio, "Effects of subclinical thyroid dysfunction on the heart," Annals of Internal Medicine, vol. 137, no. 11, pp. 904-914, 2002.

[38] M. Kivipelto, E. L. Helkala, M. P. Laakso et al., "Midlife vascular risk factors and Alzheimer's disease in later life: longitudinal, population based study," BMJ (Clinical research ed.), vol. 322, no. 7300, pp. 1447-1451, 2001.

[39] J. Hardy and D. J. Selkoe, "The amyloid hypothesis of Alzheimer's disease: progress and problems on the road to therapeutics," Science, vol. 297, no. 5580, pp. 353-356, 2002, Retraction in: Science. 2002 Nov 1;298(5595):962-4; author reply 962-4.

[40] K. J. Tsai, Y. C. Tsai, and C. K. Shen, "G-CSF rescues the memory impairment of animal models of Alzheimer's disease," The Journal of Experimental Medicine, vol. 204, no. 6, pp. 12731280, 2007.

[41] E. Hogervorst, F. Huppert, F. E. Matthews, and C. Brayne, "Thyroid function and cognitive decline in the MRC Cognitive Function and Ageing Study," Psychoneuroendocrinology, vol. 33, no. 7, pp. 1013-1022, 2008. 
[42] T. Ren, Y. Gao, Y. Qiu et al., "Gut microbiota altered in mild cognitive impairment compared with normal cognition in sporadic Parkinson's disease," Frontiers in Neurology, vol. 11, p. 137, 2020.

[43] D. Cuervo-Zanatta, J. Garcia-Mena, and C. Perez-Cruz, "Gut microbiota alterations and cognitive impairment are sexually dissociated in a transgenic mice model of Alzheimer's disease," Journal of Alzheimer's disease, vol. 82, no. s1, pp. S195-s214, 2021.

[44] A. Ticinesi, C. Tana, A. Nouvenne, B. Prati, F. Lauretani, and T. Meschi, "Gut microbiota, cognitive frailty and dementia in older individuals: a systematic review," Clinical Interventions in Aging, vol. 13, pp. 1497-1511, 2018.

[45] F. Fransen, A. A. van Beek, T. Borghuis et al., "Aged gut microbiota contributes to systemical inflammaging after transfer to germ-free mice," Frontiers in Immunology, vol. 8, p. 1385, 2017.

[46] H. E. Jakobsson, A. M. Rodríguez-Piñeiro, A. Schütte et al., "The composition of the gut microbiota shapes the colon mucus barrier," EMBO Reports, vol. 16, no. 2, pp. 164-177, 2015.

[47] S. M. Finegold, “_Desulfovibrio_species are potentially important in regressive autism," Medical Hypotheses, vol. 77, no. 2, pp. 270-274, 2011.

[48] I. Mukhopadhya, R. Hansen, E. M. El-Omar, and G. L. Hold, "IBD-what role do Proteobacteria play?," Nature Reviews. Gastroenterology \& Hepatology, vol. 9, no. 4, pp. 219-230, 2012.

[49] Y. Qian, X. Yang, S. Xu et al., "Detection of microbial 16S rRNA gene in the blood of patients with Parkinson's disease," Frontiers in Aging Neuroscience, vol. 10, p. 156, 2018.

[50] G. Di Paolo and T. W. Kim, "Linking lipids to Alzheimer's disease: cholesterol and beyond," Nature Reviews. Neuroscience, vol. 12, no. 5, pp. 284-296, 2011.

[51] J. C. Lambert, C. A. Ibrahim-Verbaas, D. Harold et al., "Metaanalysis of 74,046 individuals identifies 11 new susceptibility loci for Alzheimer's disease," Nature Genetics, vol. 45, no. 12, pp. 1452-1458, 2013.

[52] G. W. Beecham, K. Hamilton, A. C. Naj et al., "Genome-wide association meta-analysis of neuropathologic features of Alzheimer's disease and related dementias," PLoS Genetics, vol. 10, no. 9, article e1004606, 2014.

[53] R. Sims, S. J. van der Lee, A. C. Naj et al., "Rare coding variants in _PLCG2_,_ABI3_, and _TREM2_ implicate microglialmediated innate immunity in Alzheimer's disease," Nature Genetics, vol. 49, no. 9, pp. 1373-1384, 2017.

[54] J. Han, Y. Jiang, Z. Li, V. V. Kravchenko, and R. J. Ulevitch, "Activation of the transcription factor MEF2C by the MAP kinase p38 in inflammation," Nature, vol. 386, no. 6622, pp. 296-299, 1997.

[55] B. P. Fairfax, P. Humburg, S. Makino et al., "Innate immune activity conditions the effect of regulatory variants upon monocyte gene expression," Science, vol. 343, no. 6175, p. 1246949, 2014.

[56] S. Ghaisas, J. Maher, and A. Kanthasamy, "Gut microbiome in health and disease: linking the microbiome-gut-brain axis and environmental factors in the pathogenesis of systemic and neurodegenerative diseases," Pharmacology \& Therapeutics, vol. 158, pp. 52-62, 2016.

[57] S. S. Yarandi, D. A. Peterson, G. J. Treisman, T. H. Moran, and P. J. Pasricha, "Modulatory effects of gut microbiota on the central nervous system: how gut could play a role in neuropsychiatric health and diseases," Journal of Neurogastroenterology and Motility, vol. 22, no. 2, pp. 201-212, 2016.

[58] P. Tognini, "Gut microbiota: a potential regulator of neurodevelopment," Frontiers in Cellular Neuroscience, vol. 11, p. 25, 2017.

[59] J. K. Nicholson, E. Holmes, J. Kinross et al., "Host-gut microbiota metabolic interactions," Science, vol. 336, no. 6086, pp. 1262-1267, 2012.

[60] S. Holmqvist, O. Chutna, L. Bousset et al., "Direct evidence of Parkinson pathology spread from the gastrointestinal tract to the brain in rats," Acta Neuropathologica, vol. 128, no. 6, pp. 805-820, 2014

[61] S. Jangi, R. Gandhi, L. M. Cox et al., "Alterations of the human gut microbiome in multiple sclerosis," Nature Communications, vol. 7, no. 1, p. 12015, 2016.

[62] T. G. Dinan and J. F. Cryan, "Gut instincts: microbiota as a key regulator of brain development, ageing and neurodegeneration," The Journal of Physiology, vol. 595, no. 2, pp. 489-503, 2017.

[63] D. G. Kim, A. Krenz, L. E. Toussaint et al., "Non-alcoholic fatty liver disease induces signs of Alzheimer's disease (AD) in wildtype mice and accelerates pathological signs of $\mathrm{AD}$ in an $\mathrm{AD}$ model," Journal of Neuroinflammation, vol. 13, no. 1, p. 1, 2016.

[64] Y. Zhang, S. Lu, Y. Yang et al., "The diversity of gut microbiota in type 2 diabetes with or without cognitive impairment," Aging Clinical and Experimental Research, vol. 33, no. 3, pp. 589-601, 2021.

[65] Y. X. Luo, M. L. Sun, P. L. Shi, P. Liu, Y. Y. Chen, and X. Peng, "Research progress in the relationship between Veillonella and oral diseases," Hua xi kou qiang yi xue za zhi = Huaxi kouqiang yixue zazhi = West China journal of stomatology, vol. 38, no. 5, pp. 576-582, 2020.

[66] J. K. Actor, Elsevier's Integrated Review Immunology and Microbiology (Second Edition), Elsevier, 2012.

[67] M. J. During, L. Cao, D. S. Zuzga et al., "Glucagon-like peptide1 receptor is involved in learning and neuroprotection," Nature Medicine, vol. 9, no. 9, pp. 1173-1179, 2003.

[68] B. Dalile, L. Van Oudenhove, B. Vervliet, and K. Verbeke, “The role of short-chain fatty acids in microbiota-gut-brain communication," Nature Reviews. Gastroenterology \& Hepatology, vol. 16, no. 8, pp. 461-478, 2019.

[69] R. Chen, Y. Xu, P. Wu et al., "Transplantation of fecal microbiota rich in short chain fatty acids and butyric acid treat cerebral ischemic stroke by regulating gut microbiota," Pharmacological Research, vol. 148, p. 104403, 2019.

[70] J. Sun, J. Xu, Y. Ling et al., "Fecal microbiota transplantation alleviated Alzheimer's disease-like pathogenesis in APP/PS1 transgenic mice," Translational Psychiatry, vol. 9, no. 1, p. 189, 2019.

[71] V. R. Figliuolo, L. M. dos Santos, A. Abalo et al., "Sulfatereducing bacteria stimulate gut immune responses and contribute to inflammation in experimental colitis," Life Sciences, vol. 189, pp. 29-38, 2017.

[72] S. Cheng, B. Han, M. Ding et al., "Identifying psychiatric disorder-associated gut microbiota using microbiota-related gene set enrichment analysis," Briefings in Bioinformatics, vol. 21, no. 3, pp. 1016-1022, 2020.

[73] C. A. Simpson, C. Diaz-Arteche, D. Eliby, O. S. Schwartz, J. G. Simmons, and C. S. M. Cowan, "The gut microbiota in anxiety and depression - a systematic review," Clinical Psychology Review, vol. 83, p. 101943, 2021. 